\title{
Avaliação da ansiedade e do viés de atenção no canal visual para estímulos emocionais em uma amostra não clínica
}

\author{
Wilson Vieira Melo \\ Instituto Brasileiro de Gestão de Negócios \\ WP - Centro de Psicoterapia Cognitivo-Comportamental \\ Alcyr Alves de Oliveira Junior \\ Universidade Federal de Ciências da Saúde de Porto Alegre \\ Marjana da Silva Peixoto \\ Procuradoria Regional da República da $4^{\underline{a}}$ Região \\ Lisiane Bizarro Araujo \\ Universidade Federal do Rio Grande do Sul
}

\begin{abstract}
Resumo
A ansiedade vem sendo estudada como fator capaz de influenciar a atenção visual seletiva em experimentos como a Visual Probe Detection Task (VPDT). Este estudo objetivou comparar o viés de atenção para imagens emocionais em pessoas com diferentes níveis de ansiedade de traço e estado usando a VPDT. Oitenta e dois estudantes universitários responderam ao IDATE. Na VPDT, utilizaram-se 12 pares de imagens com alto nível de ativação e valência negativa, pareadas com imagens controles neutras, do International Affective Picture System (IAPS). Os estímulos eram apresentados durante $200 \mathrm{~m}$. O viés de atenção não foi diferente entre os participantes com baixos e altos níveis de ansiedade de traço e estado. Tais resultados indicaram que elas não são importantes para o viés de atenção para estímulos negativos com altos níveis de ativação. Além disso, é possível que o viés de atenção para estímulos ansiogênicos seja observado apenas em populações clínicas.
\end{abstract}

Palavras-chave: ansiedade; Visual Probe Detection Task; atenção seletiva.

\begin{abstract}
Assessment of anxiety and attentional bias in the visual channel toward emotional stimuli in a non-clinical sample. Anxiety has been observed as a factor capable to influence the visual selective attention in experiments such as the Visual Probe Detection Task (VPDT). This study aimed to compare the attentional bias to emotional images in people with different levels of trait and state anxiety using a VPDT. Eighty-two undergraduate students performed VPDT and answered the State and Trait Anxiety Inventory. In VPDT, 12 pairs of images from International Affective Picture System (emotional image with high arousal level and negative valence/ and one control, neutral) were presented during $200 \mathrm{~m}$. There was no attentional bias difference between the participants neither with low nor high levels of trait or state of anxiety. These results indicated that trait or state anxiety might not be important to attentional bias to negative and arousing visual stimuli. It is possible that just clinical populations with anxiety disorders often show attentional bias to anxiogenic stimuli.

Keywords: anxiety; Visual Probe Detection Task; selective attention.
\end{abstract}

$\mathrm{O}$ viés de atenção para estímulos percebidos como ameaçadores, e consequentemente ansiogênicos, parece estar envolvido nos quadros de ansiedade (Kristensen, 2005; Mathews \& MacLeod, 1986; Mohlman \& Gorman, 2005). Tais estímulos podem exigir prioridade dos recursos da atenção uma vez que a ansiedade está ligada a mecanismos de sobrevivência (Estes \& Verges, 2008). Entretanto, tal viés refere-se a estímulos relacionados a ameaças específicas (RuizCaballero \& Bermúdez, 1997), isto é, fóbicos a cobras têm viés para estímulos como serpentes, cordões ou listras (Teachman, Gregg, \& Woody, 2001; Teachman \& Woody, 2004), bem como para um dependente de tabaco o viés estará relacionado a pistas relacionadas ao cigarro (Lopes, Peuker, \& Bizarro, 2008). Tal fenômeno é observado em diversos estudos, apesar de existirem dúvidas acerca de como exatamente e de que forma tais aspectos podem estar presentes (Ruiz-Caballero \& Bermúdez, 1997).

Assim como a ansiedade é um mecanismo adaptativo de sobrevivência dos organismos (Cloninger, 1994), a atenção seletiva também parece ter tal função, seja pela vigilância ou pela dificuldade em deixar de investir os recursos da atenção de estímulos de ameaça. A atenção seletiva pode catalisar os recursos de forma a priorizar as informações mais relevantes 
para o indivíduo. Existem evidências de que o processamento da atenção pode apresentar alguma seletividade para estímulos como, por exemplo, animais peçonhentos (Lipp \& Derakshan, 2005). Estes animais são ameaça para a sobrevivência do indivíduo e talvez por isso a atenção acabe priorizando tais estímulos em detrimento de outros menos ameaçadores. Outros estudos sustentam que os estados emocionais ansiosos influenciam no direcionamento de recursos atencionais para estímulos ameaçadores (Mogg, Mathews, Bird, \& MacgregorMorris, 1990). Tais alterações do funcionamento atencional podem ter um importante impacto no processamento de informação nas psicopatologias, possivelmente participando de alguma forma de sua gênese e manutenção.

Nos transtornos de ansiedade, uma distorção das informações relacionada ao perigo pode atrair uma quantidade elevada de recursos da atenção, exigindo um maior desgaste no processamento da informação, uma vez que ativa as memórias de ameaças dos indivíduos ansiosos (Puliafico \& Kendall, 2006). Apesar de diversos estudos terem sido produzidos com a finalidade de demonstrar a existência do viés de atenção nos transtornos de ansiedade, tais experimentos comumente são construídos com estímulos específicos para as psicopatologias de suas amostras (Keogh, Dillon, Georgiou, \& Hunt, 2001; Li, Wang, Poliakoff, \& Luo, 2007; Peretti, 1998; Ruiz-Caballero \& Bermúdez, 1997). Dentre os estímulos utilizados na construção dos experimentos estão imagens com alto grau de ativação e valência de agradabilidade negativa (Cacioppo, Petty, Feinstein, \& Jarvis, 1996; Hamm, Greenwald, Bradley, \& Lang, 1993; Lang \& Davis, 2006; Lang, Levin, Miller, \& Kozak, 1983; Lang, McTeague, \& Cuthbert, 2006; Lavy \& Hout, 1994; Miller et al., 1987). Apesar de alguns estudos utilizarem faces emocionais como estímulos (Fox, Russo, \& Dutton, 2002; Mogg, Garner, \& Bradley, 2007; Putman, Hermans, \& van Honk, 2006) ou até mesmo palavras (Eckhardt \& Cohen, 1997; Hester, Dixon, \& Garavan, 2006; Peretti, 1998), outros têm sido construídos com base nas imagens do Interational Affective Picture System - IAPS (Lang, Bradley, \& Cuthbert, 1999). Tais estudos costumam adotar como critério para seleção dos estímulos, exatamente o nível de ativação e valência (Koster, Crombez, Verschuere, \& De Houwer, 2004; Liu, Qian, Zhou, \& Wang, 2006).

Uma questão importante é o quanto um estímulo com valência emocional negativa e alto nível de ativação pode eliciar o viés de atenção em uma amostra sem nenhuma psicopatologia específica (Bar-Haim, Lamy, Pergamin, Bakermans-Kranenburg, \& van IJzendoorn, 2007). Alguns estudos nessa área utilizam a Visual Probe Detection Task, para medir o viés de atenção para imagens emocionalmente valentes (Asmundson \& Stein, 1994; Dalgleish et al., 2003; Mogg \& Bradley, 1998; Taghavi, Neshat-Doost, Moradi, Yule, \& Dalgleish, 1999; Vasey, Daleiden, Williams, \& Brown, 1995). A tarefa consiste em uma sequência na qual duas imagens são apresentadas em uma tela de computador por curtos intervalos de tempo. A tarefa mede o tempo que o indivíduo leva para responder ao aparecimento de uma seta quando ela substitui a imagem relevante para a sua patologia, bem como quando substitui imagens controle (BarHaim et al., 2007). Este método de investigação da atenção seletiva será também empregado neste estudo.
Contudo, o que difere o presente trabalho das muitas publicações na área é que as imagens alvo não são específicas para nenhuma patologia a priori e nem os participantes possuem qualquer psicopatologia. Tal método foi adotado a fim de se averiguar se os estímulos utilizados, quais sejam, imagens com alto nível de ativação e valência negativa, seriam capazes de eliciar uma resposta enviesada da atenção seletiva em uma amostra de sujeitos sem nenhuma psicopatologia identificada.

Indivíduos clinicamente ansiosos demonstram um padrão de processo seletivo que opera de modo a favorecer a codificação de informação ameaçadora (Williams, Mathews, \& MacLeod, 1996). Existe a necessidade de conhecer mais acerca do uso de estímulos alvos na Visual Probe Detection Task com uma valência negativa e um alto grau de ativação em uma população com baixos níveis de ansiedade. Além disso, o tempo de exposição das imagens na maioria dos estudos geralmente é de $500 \mathrm{~m}$ ou mais. Contudo, com esta duração de exposição, a atenção pode ser mantida conscientemente no estímulo alvo ou até mesmo esquivar-se dele (Arguedas, Green, Langdon, \& Coltheart, 2006; Bradley, Mogg, \& Lee, 1997; Erthal et al., 2005; Li, VanRullen, Koch, \& Perona, 2002). Com um tempo de exposição mais curto tal como $200 \mathrm{~m}$, a atenção dos indivíduos com níveis de ansiedade baixa e alta pode se comportar de modo diferente, uma vez que eles não teriam condições de decidir voluntariamente para qual estímulo atentar. $\mathrm{O}$ objetivo deste trabalho foi o de verificar se estímulos visuais com valência negativa e que produzem alto nível de ativação quando apresentados por $200 \mathrm{~m}$ podem induzir viés na atenção visual de sujeitos com diferentes níveis de ansiedade de traço e de estado, mesmo sem nenhuma psicopatologia específica diagnosticada.

\section{Método}

\section{Participantes}

A amostra foi selecionada por conveniência, e composta devido aos critérios de exclusão por 82 estudantes de uma universidade privada do sul do Brasil $(M=14$ anos de escolaridade, $D P=1,9)$. Ademais, eram de lateralidade exclusivamente destra, com idades entre 18 e 26 anos $(M=22$ anos, $D P=2,3)$, sendo que 41 eram do sexo masculino.

Foram excluídos seis participantes que eram de lateralidade sinistra, haviam consumido qualquer substância psicotrópica ilícita nos últimos 12 meses, haviam utilizado cafeína ou nicotina nas últimas duas horas ou que tinham ingerido bebida alcoólica nas últimas 24 horas. Além disso, também foram excluídos das análises todos aqueles que faziam uso regular de qualquer medicamento de uso contínuo, com exceção de anticoncepcional ( $n=11)$, declararam ter sensibilidade a imagens que envolvessem cenas de violência ou sangue $(n=9)$, ou que tinham qualquer dificuldade visual não corrigida com uso de lente ou óculos ( $n$ $=1$ ) ou ainda que pontuaram mais do que sete pontos na escala Self Report Questionnaire - SRQ-20 $(n=15)$. Ademais foram excluídos dois participantes por falha do equipamento na hora da aplicação $(n=2)$.

A maioria dos participantes era solteira $(89,3 \%)(n=75)$, trabalhava durante o dia e estudava à noite $(76,2 \%)(n=64)$. 
A avaliação do tipo de ritmo de atividade circadiana de sono e vigília indicou que 9,5\% $(n=8)$ eram matutinos, $23,8 \%(n$ =20) foram avaliados como vespertinos e $66,7 \%(n=56)$ demonstraram ser indiferentes quanto ao ritmo circadiano de sono e vigília predominante.

\section{Instrumentos}

Para caracterizar a amostra e identificar fatores de exclusão, foi utilizada uma ficha de dados sociodemográficos e biomédicos que incluía uma avaliação do nível socioeconômico, anos de escolaridade, se trabalhava e estudava ou se apenas estudava, além do estado civil, histórico de doenças crônicas e ritmo circadiano de sono/vigília.

Para avaliação dos critérios de exclusão da amostra foi utilizada a versão em português da Self Report Questionnaire - SRQ (Harding et al., 1980). O SRQ é um questionário de identificação de distúrbios psiquiátricos em nível de atenção primária que já foi validado para a população brasileira (Mari $\&$ Willians, 1986). Este instrumento é composto por 20 questões elaboradas para detecção de sofrimento mental (Borges, Jardim, Silva Filho, \& Silva, 1997; Santos, de Araújo, \& de Oliveira, 2009). O ponto de corte utilizado tem sido de sete respostas afirmativas (Smaira, Kerr-Corrêa, \& Contel, 2003).

Com o intuito de se avaliar os tipos de ansiedade de traço e de estado foi utilizado o Inventário de Ansiedade Traço-Estado - IDATE (Spielberger, Gorsuch, Lushene, Vagg, \& Jacobs, 1983). Tal instrumento é composto de duas escalas distintas de autorrelato, com vinte questões cada, elaboradas para medir dois conceitos diferentes de ansiedade (A-estado) e traço de ansiedade (T-traço) (Spielberger, Gorsuch, \& Lushene, 2003).
Para avaliar o viés de atenção visual seletiva, foi aplicada a Visual Probe Detection Task (MacLeod, Mathews, \& Tata, 1986). Tal tarefa consiste na apresentação de uma cruz no centro da tela e é orientado ao sujeito que fixe seu olhar nela. Após, são apresentadas simultaneamente duas imagens lado a lado por 200 $\mathrm{m}$, sendo uma delas o alvo e outra o controle. Após esta primeira etapa, é apresentada uma seta medindo $1,5 \mathrm{~cm}$, apontada para cima ou para baixo, que aparece no lugar de uma das imagens anteriormente apresentadas e o tempo de reação também é medido em milissegundos. A tarefa do sujeito é indicar para onde a seta está apontando. O viés é medido a partir do tempo de reação, uma vez que tempos menores quando a imagem alvo é substituída pela seta indica que o indivíduo estava olhando para aquele ponto naquele momento. Essa tarefa foi construída no software E-prime 2 (Psychology Software Tools, Inc. EUA) e apresentada em um monitor colorido de 17 polegadas, com resolução de 1280 x 1024, que era conectado a um laptop. Os dois teclados utilizados eram um deles convencional adaptado para a tarefa, e outro apenas numérico, ambos conectados ao laptop. As imagens tinham $95 \mathrm{~mm}$ de altura por $130 \mathrm{~mm}$ de largura quando expostas no monitor, enquanto a distância entre as bordas internas do par de imagens era de $30 \mathrm{~mm}$. Optou-se pelo uso de uma tarefa mais curta, isto é, com menor número de tentativas (48) do que as que vinham sendo utilizadas até então na literatura, bem como com um tempo de exposição menor do que os que vêm sendo empregados em estudos com metodologia similar $(200 \mathrm{~m})$. A apresentação do formato da tarefa é apresentada na Figura 1.

As imagens alvo e controle que compuseram esta tarefa eram oriundas do IAPS - International Affective Picture System (Lang

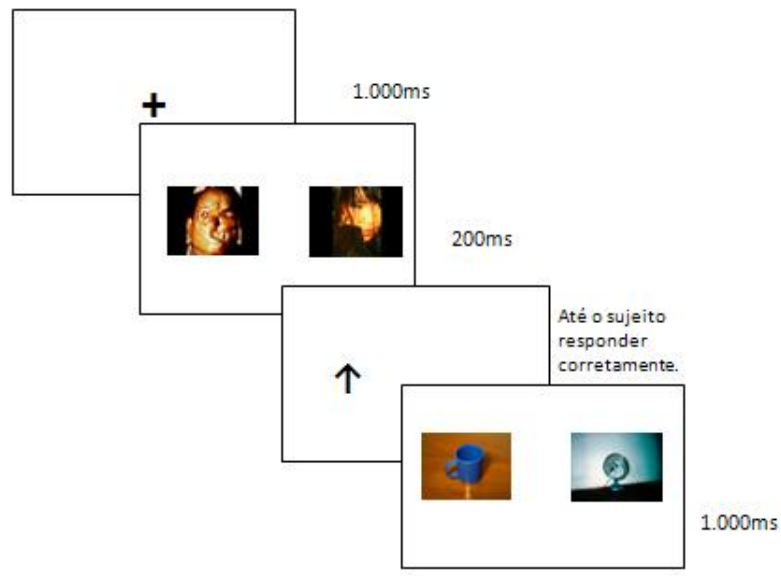

Figura 1. Apresentação da Visual Probe Detection Task.

et al., 1999). Este banco inclui centenas de fotografias coloridas de alta resolução que representam vários aspectos da vida real (esportes, moda, paisagens, violência, etc.) capazes de induzir estados emocionais que podem ser facilmente apresentados no contexto experimental do laboratório permitindo, desta forma, um controle preciso sobre o momento e a duração da exposição (Bradley \& Lang, 2000). Para a pré-seleção de 60 imagens, foram adotados os critérios de alto grau de ativação e valência negativa. Este banco de imagens fornece um conjunto normativo de avaliações emocionais para um grande número de imagens, já normatizado para a população brasileira. Após essa primeira etapa, seis juízes especialistas fizeram uma avaliação cega, isto é, sem o conhecimento da avaliação dos demais. Os juízes avaliaram individualmente e as categorizaram em uma escala entre muito ou pouco característico do funcionamento cognitivo de pacientes com transtorno de ansiedade generalizada. A partir da categorização dos juízes às imagens, construiu-se uma lista com as 12 imagens com maior nível de ativação de ansiedade de acordo com a escala. A lista de imagens controle também foi obtida do IAPS. Essas imagens foram selecionadas pelo seu 
baixo nível de ativação e valência neutra. Foram escolhidas as mais próximas em termos de configuração das cores e formas para compor a lista de imagens controle.

\section{Procedimentos}

Os participantes foram recrutados junto à universidade através de convite direto em sala de aula e através de lista de e-mails cedidas pelos coordenadores de cursos e professores. A participação no estudo era totalmente voluntária e a coleta de dados ocorreu sempre no período entre as $18: 00 \mathrm{~h}$ e as $22: 30 \mathrm{~h}$.

A coleta de dados ocorreu de forma individual, sempre na mesma sala, com aproximadamente $12 \mathrm{~m}^{2}$, e com o mesmo computador e monitor a fim de se minimizar possíveis interferências na execução das tarefas experimentais. A sala possuía iluminação adequada e baixa interferência acústica, bem como espaço para organização do material e conforto do participante e pesquisadores. $\mathrm{O}$ encontro era individual de aproximadamente 45 minutos para o preenchimento dos instrumentos que compõem o protocolo de pesquisa do estudo.

Diante da concordância em participar da pesquisa, documentada pela assinatura do Termo de Consentimento Livre e Esclarecido, e após a leitura do termo de participação onde foram apresentados os critérios de exclusão do estudo, os participantes preencheram o Self Report Questionnaire-SRQ-20. Em caso de pontuação acima de sete pontos neste instrumento ou desistência devido aos critérios de exclusão os sujeitos foram dispensados de participar do estudo. Aqueles que em condições de participar foram submetidos ao protocolo de pesquisa.

Os instrumentos foram aplicados sempre na mesma ordem iniciando pelo IDATE para avaliar os níveis de ansiedade de traço e de estado de cada indivíduo. O instrumento foi aplicado sempre antes da tarefa experimental para não sofrer uma possível influência das mesmas nos resultados avaliados pela escala de ansiedade.

Após a aplicação do instrumento de avaliação da ansiedade os sujeitos foram submetidos a Visual Probe Detection Task. A tela do computador era elevada até a altura dos olhos do participante sentado e posicionada a uma distância padrão de $60 \mathrm{~cm}$ do seu rosto. O participante foi orientado a responder seguindo orientações apresentadas na tela do computador bem como cinco tentativas de teste para familiarização com o instrumento. As instruções solicitavam que os participantes olhassem fixamente para uma cruz de fixação apresentada no centro da tela por 1000 milissegundos (ms). Em seguida, deveriam apertar com o dedo indicador um botão no teclado numérico onde o " 8 " representava seta para cima e o "2" para baixo, indicando o sentido da seta que era apresentada ora à direita e ora à esquerda do campo visual na tela do computador. Doze pares de imagens alvo e controle foram apresentados com um tempo de exposição (TE) de $200 \mathrm{~m}$ antes da apresentação do indicador (seta para cima ou para baixo). Os pares eram repetidos quatro vezes sendo que cada imagem apareceu duas vezes do lado esquerdo do monitor e duas vezes do lado direito do monitor, uma vez com a seta apontando para cima e outra para baixo, totalizando 48 tentativas em ordem randomizada. $\mathrm{O}$ indicador aparecia no local tanto das imagens relacionadas à ansiedade como das não relacionadas, com frequência igual e com a mesma quantidade de apresentação da seta para cima como da seta para baixo (Field, Mogg, Zatteler, \& Bradley, 2004).

Após a apresentação da seta e a resposta do participante, foram apresentados pares de imagens de objetos (baixo grau de ativação e valência neutra) por $3000 \mathrm{~m}$ a fim de minimizar as chances de um efeito cumulativo de ativação de ansiedade na execução da tarefa e impedir que o sujeito emita uma resposta automática por impulso.

Ao final da Visual Probe Detection Task, foi solicitado aos participantes que avaliassem através de uma escala o quão ansiogênicos ou neutros eram os estímulos-alvo e os controles a fim de se averiguar se os estímulos de fato eram apropriados para esta amostra. As imagens foram exibidas uma a uma por $2.000 \mathrm{~m}$ cada, na tela do computador para que pudessem ser avaliadas pelos participantes, sendo a ordem de apresentação aleatória. Nesse momento, foram inseridas outras 10 imagens oriundas do IAPS, com ativação baixa ou alta e valência positiva ou neutra, de forma que pudessem ser bastante diferentes dos estímulos utilizados na tarefa.

Esta pesquisa está de acordo com os aspectos éticos de pesquisas com seres humanos, segundo resolução $n^{0}$ 016/2000 (CFP, 2000) e foi aprovada pelo Comitê de Ética do Instituto de Psicologia da Universidade Federal do Rio Grande do Sul - UFRGS.

\section{Análise dos dados}

Tanto a escala de traço $(p=0,298)$ quanto a de estado ( $p$ $=0,128)$ apresentaram evidências de uma distribuição normal no teste Kolmogorov Smirnov a um nível de significância de $5 \%$. Retirou-se valores outliers, adotando-se como critério as respostas inferiores a $200 \mathrm{~m}$ de tempo de reação (TR) uma vez que a literatura indica que isso é um forte indicativo de uma resposta precipitada. Também excluiu-se respostas com TR superior a $2.000 \mathrm{~m}$, por serem indicativo de um alto grau de distratibilidade ou interferência à resposta (Dewitte, Koster, Dehouwer, \& Buysse, 2007; Koster et al., 2004). Por fim, excluiu-se respostas com dois desvios padrões acima e abaixo da média do tempo de reação do próprio sujeito, uma vez que isso denota uma resposta atípica. Calculou-se viés a partir da subtração da média do tempo de reação de cada sujeito para as imagens controle e alvo (Bradley, Mogg, \& Millar, 2000). Realizou-se análises descritivas e caracterização da amostra, seguidas de análises inferenciais para verificar as relações entre as variáveis estudadas, tais como teste $t$ para comparação entre médias de dois grupos, isto é, ansiedade alta e baixa, e também para se avaliar se a média geral era diferente de zero, ou seja, ausência de viés. Também realizou-se a comparação das médias entre o subgrupo com escores de ansiedade menores e maiores, tanto para a escala de traço quanto para a de estado.

Também se aplicou o teste de Qui-quadrado com o objetivo de verificar a associação entre os escores das escalas de ansiedade traço e estado, além do teste de correlação de Pearson.

Realizou-se uma análise de variância (ANOVA) para três e quatro grupos e, da mesma forma, para avaliar a dependência entre o tipo de ansiedade com os resultados das tarefas utilizou- 
se a análise de regressão múltipla. Os dados do tempo de reação foram logo transformados a fim de ajudar a normalizar a distribuição. Calculou-se também tamanho de efeito a fim de se avaliar a força do relacionamento entre duas variáveis em uma estimativa baseada na amostra.

Por fim, analisou-se a frequência com que os participantes avaliaram as imagens alvo, controle e positiva no que diz respeito ao nível de ativação e ao grau de agradabilidade dos estímulos.

\section{Resultados}

A média de ansiedade de traço observada foi de 39,37 pontos $(D P=10,32)$, com intervalo de escores mínimos e máximos entre 21 e 69 pontos, respectivamente. Já no que diz respeito aos escores médios da escala de ansiedade de estado a média foi de 38,1 pontos $(D P=7,46)$, onde a pontuação mínima foi de 25 e a máxima de 59 pontos. Observou-se uma associação entre os escores das escalas de ansiedade traço e estado quando divididos em baixa e alta $\left[\chi^{2}(1)=24,97, p<0,001\right]$.
Para verificar se havia viés de atenção na amostra total, foi realizado um teste $t$ para comparação da média geral $(M=8,78$, $D P=57,71)$ com zero, o que demonstrou que não havia viés $(t(81)=1,38, p=0,17, d=0,15)$.

$\mathrm{O}$ viés de atenção não foi diferente entre os participantes com baixo $(M=10,93, D P=58,61)$ e alto $(M=6,03, D P=57,25)$ níveis de ansiedade de traço nem para níveis de ansiedade de estado baixa $(M=10,88, D P=58,34)$ e alta $(M=5,5, D P=57,5)$. Não foram encontradas evidências de diferença estatisticamente significativa entre o viés médio dos sujeitos com níveis baixos e altos de ansiedade $(t(76)=0,38, p=0,704, d=0,15)$ quando relacionados com os resultados da escala de ansiedade de traço. No que diz respeito às análises comparando os níveis de ansiedade de estado, o teste $t$ para amostras independentes não mostrou um efeito de ordem significativa dos subgrupos para o viés $(t(67)=0,41, p=0,682, d=0,15)$. Os dados com as médias do viés de atenção para dois subgrupos são apresentados na Tabela 1.

Pelo teste de correlação de Pearson, há evidência de

Tabela 1

Média e desvio-padrão de viés de atenção para dois subgrupos de ansiedade de traço e estado

\begin{tabular}{lccccccc}
\hline & \multicolumn{3}{c}{ Traço } & & \multicolumn{3}{c}{ Estado } \\
\cline { 2 - 4 } \cline { 7 - 8 } Grupos & $n$ & $M$ & $D P$ & & $n$ & $M$ & $D P$ \\
\hline Baixa & 46 & 10,93 & 58,61 & & 50 & 10,88 & 58,34 \\
Alta & 36 & 6,03 & 57,25 & & 32 & 5,50 & 57,50 \\
\hline
\end{tabular}

correlação positiva entre ansiedade do tipo traço e estado a um nível de significância de 5\% $(p<0,001)$. Finalmente, foi realizada a análise de regressão com as variáveis ansiedade traço e estado, e o modelo global $(F=0,75, p=0,48)$, estado $(\beta=$ $0,14, p=0,27)$ e traço $(\beta=0,01, p=0,93)$, não foi significativo. Quando a regressão foi rodada apenas com a variável ansiedade de estado $(F=1,50, p=0,22)$, estado $(\beta=0,14, p=0,22)$, ou ainda apenas com a variável ansiedade de traço $($ Model $F=0,26$, $p=0,61)$, traço $(\beta=0,06, p=0,61)$, ainda assim não demonstrou ser significativo.

Ao final do experimento, os participantes avaliaram a adequação dos estímulos no que diz respeito ao grau de agradabilidade das imagens utilizando uma escala entre -2 (muito desagradável) a +2 (muito agradável). As imagens alvo foram consideradas desagradáveis $(M=-1,67, D P=0,62)$ e as imagens controle como sendo neutras $(M=0,04, D P=0,91) \mathrm{e}$ as positivas como agradáveis $(M=1,55, D P=0,66)$. Não houve diferença quanto à avaliação de agradabilidade das imagens entre indivíduos com traço de ansiedade baixa e alta $(t(80)=0,49, p$ $=0,63, d=4,4)$ ou estado de ansiedade baixo e alto $(t(80)=$ $0,51, p=0,61, d=4,4)$.

Já no que diz respeito à avaliação do nível de ativação de ansiedade provocado pelas imagens, os participantes julgaram as imagens alvo como sendo bastante ansiogênicas em sua maioria $(M=2,72 ; D P=1,36)$ e as imagens controle como pouco ansiogênicas $(M=0,70 ; D P=1,02)$, em uma escala de 0 (pouco ansiogênico) a 4 (muito ansiogênico). Por fim, não houve diferença na avaliação das imagens quanto ao nível de ativação entre indivíduos com traço de ansiedade baixa e alta $(t(80)=$ $0,26, p=0,79, d=-0,32$ ) ou estado de ansiedade alto ou baixo $(t(50)=-1.24, p=0,22, d=0,43)$.

\section{Discussão}

As razões para esta ausência de viés de atenção para as imagens alvo nesta amostra provavelmente se devem a um conjunto de fatores e não a um fato isolado. O uso de uma tarefa com número reduzido de tentativas pode ter influenciado no resultado e isso pode ser encarado como uma limitação do presente trabalho. Além disso, as imagens não são relacionadas especificamente a nenhuma psicopatologia. O estudo publicado por Dewitte et al. (2007) avaliando viés de atenção com a Visual Probe Detection Task para palavras com e sem especificidade demonstrou que o viés negativo foi específico apenas para palavras específicas e não para as relacionadas a ameaça de um modo geral. As imagens utilizadas, apesar de apresentarem uma valência negativa, com alto grau de ativação, não eram específicas para nenhuma psicopatologia, bem como os participantes não foram selecionados com base em uma característica cognitiva específica, tal como, por exemplo, medo de cobras. Pelo contrário, foi utilizado o SRQ-20 como um instrumento que pudesse excluir indivíduos com sintomas importantes de alguma psicopatologia em especial.

Uma vez que tal instrumento foi utilizado, isso pode ter produzido um viés de amostra, uma vez que, apesar dos estímulos terem sido avaliados pelos sujeitos como tendo uma alta ativação e valência negativa, eles podem não possuir um efeito significativo nesta amostra específica.

Outro importante aspecto a ser discutido foi o tempo de exposição dos estímulos na tarefa experimental. Apesar de a 
maioria dos estudos utilizarem um tempo de exposição de 500 $\mathrm{m}$, o tempo de $200 \mathrm{~m}$ não parece ter influenciado nos resultados, uma vez que existem tarefas experimentais que utilizam este tempo de exposição ou até mesmo menores (Erthal et al., 2005; Li et al., 2002; Mogg, Bradley, De Bono, \& Painter, 1997). O estudo de Mogg et al. (1997) demonstrou viés de atenção em três tempos de exposição (100 m, 500 m e 1.500 m), em uma amostra não clínica testada na Visual Probe Detection Task tendo palavras como estímulos. Os experimentos de Erthal et al. (2005), mostraram interferência emocional de imagens de corpos humanos mutilados do banco IAPS apresentados por $200 \mathrm{~m}$ sobre a atenção. Apesar de não existir consenso na literatura (Mathews \& Mackintosh, 1998; Mogg \& Bradley, 1998) alguns autores têm postulado que a atenção seletiva para estímulos ameaçadores é uma característica específica de populações clínicas e indivíduos com alta ansiedade (Eysenck, 1992; Williams, Watts, MacLeod, \& Mathews, 1988). Em um estudo que avaliou ansiedade em estudantes universitários foi empregada a Visual Probe Detection Task Paradigm para avaliar se o viés de atenção ou a dificuldade em desprender-se do estímulo alvo era o responsável pelos resultados nas tarefas de atenção visual seletiva (Koster et al., 2004). Os resultados apresentados nesse experimento sugerem que mais do que direcionar os recursos atencionais rapidamente para os estímulos alvo, os participantes tiveram uma dificuldade em deixar de investir tais recursos de atenção do estímulo alvo. Esse fenômeno seria o responsável pelos resultados de lentificação na resposta dos participantes de tais experimentos quando avaliados os tempos de reação para os estímulos controle, indicando que o paradigma da Visual Probe Detection Task pode gerar resultados ambíguos.

Um exemplo desse tipo de ambiguidade presente nos experimentos que utilizam esta metodologia é o de que em um estudo (Liu et al., 2006) não foi possível observar diferença na atenção entre os participantes que tinham índices de ansiedade alta e baixa. Neste trabalho, apesar de num primeiro momento ter sido observado o viés, ele foi desaparecendo gradualmente, à medida que as tentativas iam sendo disparadas. Outro trabalho que também utilizou as imagens do IAPS com o uso da Visual Probe Detection Task em uma amostra de estudantes universitários também não foi encontrado viés de atenção (Koster et al., 2004). Da mesma forma que no presente estudo, os participantes não tinham nenhuma psicopatologia específica, o que reforça a ideia de que a especificidade do conteúdo cognitivo precisa ser congruente com as características específicas dos estímulos utilizados. Apesar disso, a ansiedade é frequentemente uma variável associada ao viés de atenção.

A interferência da ansiedade sobre a atenção foi observada, por exemplo, quando sujeitos ansiosos apresentaram um aumento na quantidade de recursos atencionais dirigidos para um contexto negativo. Indivíduos com ansiedade de estado alta avaliada pelo Inventário de Ansiedade Traço-Estado (IDATE) (Spielberger et al, 1983) tiveram um maior investimento de recursos atencionais para estímulos visuais com altos níveis de ativação e valência negativa (Mercado, Carretié, Tapia, \& Gómez-Jarabo, 2006).

O fato dos estímulos possuírem características importantes tais como forte ativação emocional e valência negativa não implicam em necessariamente nas capacidades de evocar algum funcionamento enviesado da atenção seletiva. A especificidade do conteúdo cognitivo nas psicopatologias faz com que o funcionamento da atenção sofra alterações significativas (Knapp \& Beck, 2008). Entretanto, isso não quer dizer que não seja necessário se conhecer mais acerca do funcionamento de indivíduos psicologicamente saudáveis. Conhecer mais acerca destes fenômenos atencionais, principalmente nos diferentes canais perceptivos, pode ser de fundamental importância para se desenvolver novas técnicas de tratamento dos transtornos de ansiedade. Além disso, pode-se até mesmo desenvolver ferramentas que aperfeiçoem processos de aprendizagem e de utilização dos recursos atencionais em populações não clínicas, uma vez que a ansiedade não é exclusiva das psicopatologias.

\section{Referências}

Arguedas, D., Green, M. J., Langdon, R., \& Coltheart, M. (2006). Selective attention to threatening faces in delusion-prone individuals. Cognitive Neuropsychiatry, 11(6), 557-575. doi:10.1080/13546800500305179

Asmundson, G. J. G., \& Stein, M. B. (1994). Selective processing of social threat in patients with generalized social phobia: evaluation using a dot-probe paradigm. Journal of Anxiety Disorders, 8(2), 107-117. doi:10.1016/08876185(94)90009-4

Bar-Haim, Y., Lamy, D., Pergamin, L., Bakermans-Kranenburg, M. J., \& van IJzendoorn, M. H. (2007). Threat-related attentional bias in anxious and nonanxious individuals: a meta-analytic study. Psychological Bulletin, 133(1), 1-24. doi:10.1037/0033-2909.133.1.1

Borges, L. H., Jardim, S. R., Silva Filho, J. F., \& Silva, M. G. R. (1997). Uso do self reporting questionnaire (SRQ-20) em estudos sobre a saúde mental dos trabalhadores. Jornal Brasileiro de Psiquiatria, 46(9), 483-486.

Bradley, B. P, Mogg, K., \& Lee, S. C. (1997). Attentional biases for negative information in induced and naturally occurring dysphoria. Behaviour Research and Therapy, 35(10), 911-927.

Bradley, B. P., Mogg, K., \& Millar, N. H. (2000). Covert and overt orienting of attention to emotional faces in anxiety. Cognition \& Emotion, 14(6), 789-808. doi:10.1080/02699930050156636

Bradley, M. M., \& Lang, P. J. (2000). Measuring emotion: behavior, feeling and physiology. In R. Lane \& L. Nadel (Orgs.), Cognitive neuroscience of emotion (pp. 242-276). Nova Iorque: Oxford University Press.

Cacioppo, J. T., Petty, R. E., Feinstein, J. A., \& Jarvis, W. B. G. (1996). Dispositional differences in cognitive motivation: the life and times of individuals varying in need for cognition. Psychological Bulletin, 119(2), 197-253.

Conselho Federal de Psicologia. (2000). Resolução para pesquisa com seres humanos. Resolução 016/2000. Brasília: Conselho Federal de Psicologia.

Cloninger, C. R. (1994). The genetic structure of personality and learning: a phylogenetic model. Clinical Genetics, 46(1), 124-137.

Dalgleish, T., Taghavi, R., Neshat-Doost, H., Moradi, A., Canterbury, R., \& Yule, W. (2003). Patterns of processing bias for emotional information across clinical disorders: a comparison of attention, memory, and prospective cognition in children and adolescents with depression, generalized anxiety, and posttraumatic stress disorder. Journal of Clinical Child and Adolescent Psychology, 32, 10-21.

Dewitte, M., Koster, E., Dehouwer, J., \& Buysse, A. (2007). Attentive processing of threat and adult attachment: a dot-probe study. Behaviour Research and Therapy, 45(6), 1307-1317. doi:10.1016/j.brat.2006.11.004

Eckhardt, C. I., \& Cohen, D. J. (1997). Attention to anger-relevant and irrelevant stimuli following naturalistic insult. Personality and Individual Differences, 
23(4), 619-629. doi:10.1016/S0191-8869(97)00074-3

Erthal, F. S., de Oliveira, L., Mocaiber, I., Pereira, M. G., Machado-Pinheiro, W., Volchan, E., \& Pessoa, L. (2005). Load-dependent modulation of affective picture processing. Cognitive and Affective Behavior Neuroscience, 5(4), 388-395.

Estes, Z., \& Verges, M. (2008). Freeze or flee? Negative stimuli elicit selective responding. Cognition, 108(2), 557-565.

Eysenck, M. W. (1992). Anxiety: the cognitive perspective. Hove, England: Erlbaum.

Field, M., Mogg, K., Zatteler, J., \& Bradley, B. (2004). Attentional biases for alcohol cues in heavy and light social drinkers: the roles of initial orienting and maintained attention. Psychoparmacology, 176, 88-93.

Fox, E., Russo, R., \& Dutton, K. (2002). Attentional bias for threat: evidence for delayed disengagement from emotional faces. Cognition \& Emotion, 16(3), 355-379.

Hamm, A. O., Greenwald, M. K., Bradley, M. M., \& Lang, P. J. (1993). Emotional learning, hedonic change, and the startle probe. Journal of Abnormal Psychology, 102, 453-465.

Harding, T. W., Arango, M. V., Baltazar, J., Climent, C. E., Ibrahim, H. H. A., Ignácio, L. L., ... Wig, N. N. (1980). Mental Disorders in primary health care: a study of their frequency and diagnosis in four development countries. Psychological Medicine, 10, 231-241.

Hester, R., Dixon, V., \& Garavan, H. (2006). A consistent attentional bias for drugrelated material in active cocaine users across word and picture versions of the emotional Stroop task. Drug and Alcohol Dependence, 81(3), 251-257. doi:10.1016/j.drugalcdep.2005.07.002

Keogh, E., Dillon, C., Georgiou, G., \& Hunt, C. (2001). Selective attentional biases for physical threat in physical anxiety sensitivity. Journal of Anxiety Disorders, 15(4), 299-315.

Knapp, P., \& Beck, A. T. (2008). Cognitive therapy: foundations, conceptual models, applications and research. Revista Brasileira de Psiquiatria, 30(2), 54-64.

Koster, E. H. W., Crombez, G., Verschuere, B., \& De Houwer, J. (2004). Selective attention to threat in the dot probe paradigm: differentiating vigilance and difficulty to disengage. Behaviour Research and Therapy, 42(10), 1183-1192. doi:10.1016/j.brat.2003.08.001

Kristensen, C. H. (2005). Estresse pós-traumático: sintomatologia e funcionamento cognitivo. (Tese de Doutorado). Universidade Federal do Rio Grande do Sul, Porto Alegre.

Lang, P. J., Bradley, M. M., \& Cuthbert, B. N. (1999). International affective picture system (IAPS): Instruction manual and affective ratings. Technical Report A-4. Florida: The Center for Research in Psychophysiology.

Lang, P. J., \& Davis, M. (2006). Emotion, motivation, and the brain: reflex foundations in animal and human research. Progress in Brain Research, 156, 3-34.

Lang, P. J., Levin, D. N., Miller, G. A., \& Kozak, M. J. (1983). Fear imagery and the psychophysiology of emotion: the problem of affective response integration. Journal of Abnormal Psychology, 92, 276-306.

Lang, P. J., McTeague, L. M., \& Cuthbert, B. N. (2006). Fearful imagery and the anxiety disorder spectrum. In B. Rothbaum (Org.), Pathological anxiety: emotional processing in etiology and treatment (pp. 56-77). New York: The Guilford Press.

Lavy, E. H., \& Hout, M. A. (1994). Cognitive avoidance and attentional bias: causal relationships. Cognitive Therapy and Research, 18(2), 179-191. doi:10.1007/BF02357223

Li, F. F., VanRullen, R., Koch, C., \& Perona, P. (2002). Rapid natural scene categorization in the near absence of attention. Proceedings of the National Academy of Sciences of the United States of America, 99(14), 9596-9601.

Li, X., Wang, M., Poliakoff, E., \& Luo, Y. J. (2007). Attention to threat in high and low trait-anxious individuals: a study using extremely threatening pictorial cues. Perceptual and Motor Skills, 104(3 Pt 2), 1097-1106.
Lipp, O. V., \& Derakshan, N. (2005). Attentional bias to pictures of fear-relevant animals in a dot probe task. Emotion, 5(3), 365-369.

Liu, X., Qian, M., Zhou, X., \& Wang, A. (2006). Repeating the stimulus exposure to investigate what happens after initial selective attention to threatening pictures. Personality and Individual Differences, 40(5), 1007-1016. doi:10.1016/j.paid.2005.10.006

Lopes, F. M., Peuker, A. C., \& Bizarro, L. (2008). Viés atencional em fumantes. Psico, 39(3), 280-288.

MacLeod, C., Mathews, A., \& Tata, P. (1986). Attentional bias in emotiona disorders. Journal of Abnormal Psychology, 95, 15-20.

Mari, J., \& Willians, P. A. (1986). A validity study of a psychiatric screening questionnaire (SRQ-20) in primary care in the city of São Paulo. British Journal of Psychiatry, 148, 23-26.

Mathews, A., \& MacLeod, C. (1986). Discrimination of threat cues without awareness in anxiety states. Journal of Abnormal Psychology, 95(2), 131-138.

Mathews, A, \& Mackintosh, B. (1998). A cognitive model of selective processing in anxiety. Cognitive Therapy and Research, 22(6), 539-560. doi:10.1023/A:1018738019346

Mercado, F., Carretié, L., Tapia, M., \& Gómez-Jarabo, G. (2006). The influence of emotional context on attention in anxious subjects: neurophysiological correlates. Journal of Anxiety Disorders, 20(1), 72-84.

Miller, G. A., Levin, D. N., Kozak, M. J., Cook, E. W., McLean, I., Jr., A., \& Lang, P. J. (1987). Individual differences in imagery and the psychophysiology of emotion. Cognition and Emotion, 1, 367-390.

Mogg, K., Bradley, B. P., De Bono, J., \& Painter, M. (1997). Time course of attentional bias for threat information in non-clinical anxiety. Behaviour Research and Therapy, 35(4), 297-303.

Mogg, K., Garner, M., \& Bradley, B. P. (2007). Anxiety and orienting of gaze to angry and fearful faces. Biological Psychology, 76(3), 163-169.

Mogg, K., Mathews, A., Bird, C., \& Macgregor-Morris, R. (1990). Effects of stress and anxiety on the processing of threat stimuli. Journal of Personality and Social Psychology, 59(6), 1230-1237.

Mogg, K, \& Bradley, B. P. (1998). A cognitive-motivational analysis of anxiety. Behaviour Research and Therapy, 36(9), 809-848. doi:10.1016/S00057967(98)00063-1

Mohlman, J., \& Gorman, J. M. (2005). The role of executive functioning in CBT: a pilot study with anxious older adults. Behaviour Research and Therapy, 43(4), 447-465.

Peretti, C. S. (1998). Anxiety and cognition disorders. Encephale, 24(3), 256-259.

Puliafico, A. C., \& Kendall, P. C. (2006). Threat-related attentional bias in anxious youth: a review. Clinical Child and Family Psychology Review, 9(3-4), 162 180. doi:10.1007/s10567-006-0009-x

Putman, P., Hermans, E., \& van Honk, J. (2006). Anxiety meets fear in perception of dynamic expressive gaze. Emotion, 6(1), 94-102.

Ruiz-Caballero, J. A., \& Bermúdez, J. (1997). Anxiety and attention: is there an attentional bias for positive emotional stimuli? The Journal of General Psychology, 124(2), 194-210.

Santos, K. O., de Araújo, T. M., \& de Oliveira, N. F. (2009). Estrutura fatorial e consistência interna do Self-Reporting Questionnaire (SRQ-20) em população urbana. Cadernos de Saúde Pública, 25(1), 214-222.

Smaira, S. I., Kerr-Corrêa, F., \& Contel, J. O. (2003). Transtornos psiquiátricos e solicitações de interconsulta psiquiátrica em hospital geral: um estudo de caso-controle. Revista Brasileira de Psiquiatria, 200(25), 18-25.

Spielberger, C. D., Gorsuch, R. L., \& Lushene, R. E. (2003). IDATE: Manual do Inventário de Ansiedade traço-estado (Vol. 2). Rio de Janeiro: CEPA.

Spielberger, C. D., Gorsuch, R. L., Lushene, R. E., Vagg, P., \& Jacobs, G. A. (1983). Manual for the State-Trait Anxiety Inventory (Form Y) (selfevaluation questionnaire). Palo Alto: Consulting Psychologists Press.

Taghavi, M. R., Neshat-Doost, H. T., Moradi, A. R., Yule, W., \& Dalgleish, 
T. (1999). Biases in visual attention in children and adolescents with clinical anxiety and mixed anxiety-depression. Journal of Abnormal Child Psychology, 27(3), 215-223. doi:10.1023/A:1021952407074

Teachman, B. A., Gregg, A., \& Woody, S. (2001). Implicit processing of fearrelevant stimuli among individuals with snake and spider fears. Journal of Abnormal Psychology, 110, 226-235.

Teachman, B. A., \& Woody, S. (2004). Staying tuned to research in implicit cognition: Relevance for clinical practice with anxiety disorders. Cognitive and Behavioral Practice, 11, 149-159.

Vasey, M. W., Daleiden, E. L., Williams, L. L., \& Brown, L. M. (1995). Biased attention in childhood anxiety disorders: a preliminary study. Journal of Abnormal Child Psychology, 23, 267-279.

Williams, J. M., Mathews, A., \& MacLeod, C. (1996). The emotional Stroop task and psychopathology. Psychological Bulletin, 120(1), 3-24.

Williams, J. M. G., Watts, F. N., MacLeod, C., \& Mathews, A. (1988). Cognitive Psychology and Emotional Disorders. Nova Iorque: Wiley.

Wilson Vieira Melo, doutor em Psicologia pela Universidade Federal do Rio Grande do Sul, é professor no Instituto Brasileiro de Gestão de Negócios (IBGEN) e WP - Centro de Psicoterapia CognitivoComportamental. Endereço para correspondência: Avenida Soledade, 569 / 1206 - Torre Alfa, Bairro Três Figueiras, Porto Alegre - RS. CEP 90470-340. Telefone: (51) 3328-9622. Fax: (51) 3328-9121. Email: wilsonmelo1@gmail.com

Alcyr Alves de Oliveira Junior, doutor em Psicologia pela University of London, é professor na Universidade Federal de Ciências da Saúde de Porto Alegre. Email: alcyr@ufcspa.edu.br

Marjana da Silva Peixoto, bacharel em Estatística pela Universidade Federal do Rio Grande do Sul, é estatista responsável na Procuradoria Regional da República da 4a Região - PRR-4/MPF. Email: marjanapeixoto@ hotmail.com

Lisiane Bizarro Araujo, doutora em Psicologia pela University of London, é professora Associada na Universidade Federal do Rio Grande do Sul. Email: lisiane.bizarro@ufrgs.br 